\title{
Die Verwertung von Essigsäure durdh Chlorella im Licht
}

\author{
Von H. G. SCHLEGEL * \\ Aus dem Institut für Mikrobiologie, Göttingen \\ (Z. Naturforschg. 14 b, 246-253 [1959]; eingegangen am 15. Oktober 1958)
}

\begin{abstract}
Studies on the utilization of acetate- $2-{ }^{14} \mathrm{C}$ by Chlorella pyrenoidosa have shown that light brings about a preferential incorporation of acetate into the fatty acids of the cellular lipids. Approximately $50 \%$ of the labelled acetate-carbon added was found in the petroleumethersoluble fraction. The isotopepattern of glucose (from hydrolyzed starch) is in agreement with the function of the tricarboxylic acid-, glyoxylic acid-cycle respectively in Chlorella. The asymmetrical distribution of the labeled carbon $(\mathrm{C} 4,5,6>\mathrm{C} 1,2,3)$ in the glucose-molecule seems to indicate that there is a dilution of the dihydroxyacetone-moiety from a pool which may be fed by (trans) aldolase-reactions.
\end{abstract}

Vorangegangene Untersuchungen hatten gezeigt, daß der Umsatz organischer Substrate durch Chlorella von den Versuchsbedingungen ( \pm Licht, $\pm \mathrm{CO}_{2}$ ) quantitativ stark beeinflußt wird. Assimilationsquotienten von $\Delta \mathrm{O}_{2} /-\Delta \mathrm{CO}_{2} \approx 5$ bei Gegenwart von Essigsäure und $\mathrm{CO}_{2}$ deuteten auf eine Beeinflussung der Essigsäure-Assimilation durch Licht auch in qualitativer Hinsicht und auf einen bevorzugten Einbau des Acetats in die Fettfraktion der Zelle ${ }^{1}$. Unter Verwendung von Acetat- $2 \cdot{ }^{14} \mathrm{C}$ sollten diese Befunde überprüft werden. Darüber hinaus zielten die Versuche dieser Arbeit darauf ab, durch schrittweisen Abbau der im Licht und im Dunkeln synthetisierten Glucose das Verteilungsmuster des eingebauten Acetat-Kohlenstoffs im Glucosemolekül zu erfassen, welches Rückschlüsse auf die beteiligten Prozesse des Intermediärstoffwechsels ermöglicht. Woop und Mitarbb. ${ }^{2,9,19}$ haben die Isotopenverteilung im Glykogen der Leber mehrfach als Indikator des Intermediärstoffwechsels herangezogen und damit wesentliche Beiträge für die Funktion des Tricarbonsäurezyklus in tierischen Organen geliefert. An Grünalgen liegt bisher nur eine sehr kleine Anzahl von Untersuchungen dieser Art vor. Da an der photosynthetischen $\mathrm{CO}_{2}$-Fixierung und der Regeneration des $\mathrm{CO}_{2}$-Acceptors zahlreiche Schritte des intermediären Kohlenhydrat-Stoffwechsels beteiligt sind, dürften von experimentellen Studien über den Einbau von markierten Zuckern und organischen Säuren wesentliche Beiträge zur Aufhellung der komplexen Prozesse der Photosynthese zu erwarten sein. Gibbs und Kandler ${ }^{3}$ erfaßten die Isotopen-

* Durchgeführt mit Unterstützung der D e u t s c h e n F or . $\mathrm{sch}$ ung g e meins chaft. Dem Leiter des Department of Microbiology, School of Medicine, Western Reserve University, Cleveland, Herrn Dr. L. O. Krampitz, danke ich für die Überlassung eines Arbeitsplatzes. verteilung in Glucose, die aus Chlorella, Tabak-, Canna- und Sonnenblumenblättern nach kurzfristiger ${ }^{14} \mathrm{CO}_{2}$-Exposition im Licht gewonnen worden war, und fanden eine asymmetrische Verteilung des isotopen Kohlenstoffes über das Glucosemolekül (C $4>$ C $3>$ C 1,2,5 oder 6 ). Über einige orientierende Versuche mit markierten Acetaten berichtete Gibis $^{4}$. Da die vorliegende Untersuchung zu verwandten Ergebnissen führte, wird die Frage des $\mathrm{Zu}$ standekommens der asymmetrischen Markierung eingehend erörtert.

\section{Methoden}

Chlorella pyrenoidosa wurde herangezogen wie be. schrieben ${ }^{1}$, nach fünftägigem Wachstum bei $20^{\circ} \mathrm{C}$ (am Nordfenster und im Licht von Tageslicht-Leuchtstoffröhren) durch Zentrifugieren geerntet, gewaschen und durch 18-24 stdg. Durchströmen mit Luft im Dunkeln an Speicherstoffen verarmt. Vor dem Versuch wurden die Zellen erneut gewaschen und in $m / 30$-Phosphatpuffer $p_{\mathrm{H}} 5,6$ aufgeschwemmt. Alle Versuche wurden an reservestoffarmen Zellen durchgeführt.

Die Aufnahme von markiertem Acetat unter variierten Bedingungen ( \pm Licht, \pm Kohlendioxyd) wurde nach zwei verschiedenen Methoden verfolgt: a) Versuch 1 (Tab. 1): In 15-ml-W a rburg-Gefäßen in einer Licht - W a r b u r g - Apparatur mit Bodenbeleuchtung (ca. $1000 \mathrm{fcdls}$ am Boden der Gefäße). b) Versuch 2 (Abb. 1) : Zur kontinuierlichen Durchströmung wurde die Chlorella-Suspension in Feinsinterfiltern mit hohem Rand, die in einem gleichmäßig temperierten $\left(30^{\circ} \mathrm{C}\right)$, von der Seite belichteten (75-W-Reflektor Flodd Lamps, General Electric) Glasaquarium untergetaucht waren, von unten her mit $5 \% \mathrm{CO}_{2}$ enthaltender, bzw. $\mathrm{CO}_{2}$-freier Luft durchperlt. Nach gewissen Zeiten

1 H. G. Schlegel, Planta 47, 510 [1956].

2 H. G. Wood, N. Lifson u. V. Lorber, J. biol. Chemistry 159, 475 [1945].

3 M. Gibbs u. O. Kandler, Proc. nat. Acad. Sci. USA 43, 446 [1957].

${ }^{4}$ M. Gibss, Plant Physiol., Suppl. 32, XVII [1957]. 
wurden 5-ml-Proben der Suspension in $0,5 \mathrm{ml} 5-n$. $\mathrm{H}_{2} \mathrm{SO}_{4}$ enthaltende Zentrifugengläser auspipettiert, abzentrifugiert, mit $5 \mathrm{ml}$ 1-proz. NaCl-Lösung zweimal gewaschen, in v a n S ly k e - Röhrchen übergeführt, bei $105^{\circ} \mathrm{C}$ getrocknet und nach van Slyke und Folch ${ }^{5}$ verbrannt; das Kohlendioxyd wurde in $3,5 \mathrm{ml} 2,5-n$. $\mathrm{CO}_{2}$ freier $\mathrm{NaOH}$ aufgefangen, gewogen und die Radioaktivität nach der $\mathrm{BaCO}_{3}$-Methode bestimmt.

Zur Verfolgung des Einbaus von Acetat in verschiedene Zellfraktionen (Abschnitt II und III) wurden $7-8 \mathrm{~g}$ Algen (Naßgewicht) in $200 \mathrm{ml} \mathrm{m} / 30$-Phosphatpuffer $p_{\mathrm{H}} 5,6$ aufgeschwemmt und zu gleichen Teilen in zwei $\mathrm{F}$ e r n b a ch-Kolben (4 $l$ ) übergeführt, von denen der eine dunkel gehalten, der andere von unten her einer Lichtintensität von $2000 \mathrm{fcdls}$ ausgesetzt wurde. Beide Kolben wurden in einem Thermostaten bei $30^{\circ} \mathrm{C}$ geschüttelt. Nach $30 \mathrm{~min}$ langem Schütteln unter Durchströmung mit $5 \% \mathrm{CO}_{2}$-Luft-Gemisch wurden jedem Kolben ca. $30 \mu$ Mole Acetat- $2 \cdot{ }^{14} \mathrm{C}$ zugesetzt und Schütteln und Durchströmung für $10-30 \mathrm{~min}$ fortgesetzt. Der Einbau wurde durch Herabsetzung des $p_{\mathrm{H}}$ auf 1,0 und Eiskühlung abgebrochen; dann wurde sofort in der Kälte abzentrifugiert und einmal mit $1 \%$ $\mathrm{NaCl}$-Lösung gewaschen; die gepackten Zellen wurden bis zur Extraktion im gefrorenen Zustand aufbewahrt. Mit heißem, wäßrigem Åthanol, Aceton, einem Gemisch von Äthanol-Äther und $\ddot{A}^{2}$ ther $^{6}$ wurden die Pigmente, Fette und freien Zucker sowie deren Ester weitgehend extrahiert. Der Pigmentextrakt wurde zur Trockne eingeengt und mit trockenem Petroläther extrahiert, wobei ein beträchtlicher Teil wasserlöslicher Substanzen, darunter im wesentlichen Glucose, zurückblieben. Der Petrolätherextrakt wurde mehrmals mit Wasser gewaschen und durch Filtrieren durch eine Schicht von wasserfreiem Natriumsulfat getrocknet. Ein aliquoter Teil wurde verbrannt und die spezifische Aktivität bestimmt. Der Rest wurde nach Eindunsten mit $20 \mathrm{ml}$ einer Lösung von 5-proz. $\mathrm{KOH}$ in 50-proz., wäßrigem Methanol versetzt und durch 10-stdg. Kochen unter Rückfluß verseift. Die alkalische Lösung wurde dann mit Wasser verdünnt und mit Petroläther erschöpfend extrahiert. Diese die Kohlenwasserstoffe enthaltende Petrolätherfraktion wurde nach Waschen mit 0,1-n. $\mathrm{NaHCO}_{3}$-Lösung und Wasser durch wasserfreies $\mathrm{Na}$ triumsulfat filtriert, eingeengt, gewogen und ein aliquoter Teil zur Bestimmung der spezifischen Aktivität verbrannt. Der Rest des Unverseifbaren wurde von Petroläther an Aluminiumoxyd (aktives und deaktiviertes $1: 1)^{7}$ chromatographiert und die Aktivität der einzelnen Zonen und die spezifische Aktivität des $\beta$-Carotins bestimmt. Aus der alkalischen wäßrigen Phase wurden nach Ansäuern die Fettsäuren mit Petrol-

5 D. D. van Slyke u. J. Folch, J. biol. Chemistry 136, 509 [1940].

6 H. W. Milner, J. biol. Chemistry 176, 813 [1948].

7 T. W. Goodwin u. M. Jamikorn, Biochem. J. 62, 269 [1956].

8 I. C. Gunsalus u. M. Gibbs, J. biol. Chemistry 194, 871 [1952].

* Für die Überlassung dieses Stammes (39) danke ich Dr. J. De Moss. äther ausgeschüttelt, dann in Alkali aufgenommen und wieder in Petroläther übergetrieben; der Petroläther wurde mit 0,01-n. $\mathrm{H}_{2} \mathrm{SO}_{4}$ mehrmals gewaschen und schließlich über wasserfreiem $\mathrm{Na}_{2} \mathrm{SO}_{4}$ getrocknet. Die spezifische Aktivität dieser Fettsäurefraktion wurde sowohl in dünner Schicht als auch nach der Bariumcarbonat-Methode bestimmt.

Die fettextrahierten Algen wurden mit $4 \mathrm{ml} 1-n$. $\mathrm{H}_{2} \mathrm{SO}_{4}$ versetzt und $3 \mathrm{Stdn}$. im strömenden Dampf hydrolysiert. Aus dem Hydrolysat ließen sich durch Entsalzen an den Ionenaustauschern Duolit C-3 und Duolit A-4 und zweimalige Chromatographie mit wassergesättigtem n-Butanol an säuregewaschenem Papier Whatman Nr. 3 MM $25 \mathrm{mg}$ reine Glucose gewinnen. Die Radioautographie eines Chromatogramms der gereinig. ten Glucose (in Phenol- $\mathrm{H}_{2} \mathrm{O}$ ) zeigte, daß in dem Glucosepräparat keine verunreinigenden radioaktiven Substanzen vorhanden waren. An einem aliquoten Teil der Glucose wurde nach Verbrennung die spezifische Aktivität bestimmt.

Der Glucoseabbau erfolgte nach dem von Gunsalus und $\mathrm{GibBs}^{8}$ entwickelten und von Bernstern ${ }^{9}$ überprüften und ausgearbeiteten Verfahren der Vergärung durch den heterofermentativen Milchsäurebildner Leuconostoc mesenteroides ${ }^{*}$. Die isolierte radioaktive Glucose wurde durch reine Glucose auf $0,6-1,0 \mathrm{mMol}$ ergänzt. Frische, am Ende der log-Phase geerntete und zweimal mit physiologischer Kochsalzlösung gewaschene Zellen von Leuconostoc mesenteroides vergären Glucose $\mathrm{zu}$ $\mathrm{CO}_{2}$ (C 1), Äthanol (C 2,3) und Milchsäure (C 4, 5, 6). Kohlendioxyd wurde während der Gärung durch einen $\mathrm{N}_{2}$-Strom laufend entfernt und in $3,5 \mathrm{ml} 2,5-n$. $\mathrm{NaOH}$ aufgefangen; der Fortgang der Gärung wurde durch wiederholte Wägung der $\mathrm{CO}_{2}$-Absorptionseinheit verfolgt. Der Äthylalkohol wurde aus dem von den Zellen befreiten und alkalisch gemachten $\left(p_{H} 8,0\right)$ Gärmedium abdestilliert, mit Chromsäure zu Essigsäure oxydiert und letztere durch Wasserdampfdestillation übergetrieben, eingeengt und dem Schmidt-Abbau ${ }^{10}$ unterworfen. Aus dem Destillationsrückstand des Gärmediums wurde die Milchsäure nach Ansäuern durch Äther extrahiert und von eventuell vorhandenen flüchtigen Verbindungen durch Wasserdampfdestillation befreit. C 4 wurde mit Permanganat abgespalten ${ }^{11}$ und als $\mathrm{CO}_{2}$ in $\mathrm{NaOH}$ aufgefangen; der Acetaldehyd wurde durch Bisulfit gebunden und durch Chromsäure zu Essigsäure oxydiert (C 5,6). Durch quantitative Bestimmung der Intermediärprodukte wurde der Abbau verfolgt. Im einzel. nen wurde die Durchführungsvorschrift von SAKami ${ }^{12}$ befolgt.

Die Methode zur Aktivitätsbestimmung des $\mathrm{CO}_{2}$ ist identisch mit der, die Ormerod ${ }^{13}$ eingehend beschrieben

9 I. A. Bernstein, K. Lentz, M. Malm, P. Schambye u. H. G. Wood, J. biol. Chemistry 215, 137 [1955].

10 E. F. Phares, Arch. Biochem. Biophysics 33, 173 [1951].

11 T. E. Friedemann u. J. B. Graeser, J. biol. Chemistry 100, 291 [1933].

12 W. SAKami, Handbook of isotope tracer methods Dept. of Biochemistry, Western Reserve University, Cleveland, Ohio 1955.

13 J. G. Ormerod, Biochem. J. 64, 373 [1956]. 
hat. Alle Messungen erfolgten mit dem Tracerlab-Counter; es wurden mindestens 3000 Zerfälle gezählt; die Meßwerte wurden bezüglich des Nulleffektes und der Selbstabsorption korrigiert; die in Zerfällen pro min gemessene Aktivität wurde in Millimikro-Curies $(\mathrm{m} \mu \mathrm{C})$ umgerechnet.

\section{Ergebnisse}

\section{Vorversuche}

Die ersten Versuche zielten darauf ab, unsere früheren über die Polysaccharid-Synthese angestellten Versuche unter Anwendung der Tracer-Technik zu überprüfen und den Einfluß von Licht und Kohlendioxyd auf die Verwertung der Essigsäure zu studieren; auch sollten diese Versuche über die Bedingungen Aufschluß geben, unter denen der Einbau von markiertem Acetat zum Zwecke der Analyse einzelner Zellfraktionen zu erfolgen hat.

\begin{tabular}{|c|c|c|}
\hline & \multicolumn{2}{|c|}{$\begin{array}{c}\text { Radioaktivität } \\
\text { der Zellen }[\mathrm{m} \mu \mathrm{C}]\end{array}$} \\
& Versuch I & Versuch II \\
\hline Dunkel - $\mathrm{CO}_{2}$ & 55,6 & 55,2 \\
Dunkel $+\mathrm{CO}_{2}$ & 39,3 & 38,7 \\
Licht - $\mathrm{CO}_{2}$ & 70,5 & 61,3 \\
Licht $+\mathrm{CO}_{2}$ & 90,0 & 74,5 \\
\hline
\end{tabular}

Tab. 1. Einbau von Acetat- $2 \cdot{ }^{14} \mathrm{C}$ in Chlorella. W a r b u r g Gefäße mit Chlorella-Suspension (10,2 mg Tr.-Gew.) in $m / 15$ Phosphatpuffer $p_{\mathrm{H}} 5,5 ; 0,1 \mathrm{ml} 20$-proz. $\mathrm{KOH}$ im Zentralgefäß, bzw. $5 \% \mathrm{CO}_{2}$ in der Atmosphäre; $10 \mu$ Mole Acetat $(0,15 \mu \mathrm{C})$ aus dem Seitenarm zugefügt und nach $80 \mathrm{~min}$ mit $0,3 \mathrm{ml} 5-n \cdot \mathrm{H}_{2} \mathrm{SO}_{4}$ abgestoppt. Beleuchtungsstärke am Boden der Gefäße $1000 \mathrm{fcdls}$.

Aus Tab. 1 ist die gegenüber dem Dunkelversuch im Licht erhöhte Acetataufnahme ersichtlich. Der durch die Entziehung des Kohlendioxyds im Dunkeln beschleunigte Einbau des Acetats entspricht ganz den bereits früher durch Messung der Polysaccharid-Synthese gewonnenen Ergebnissen. Der Versuch im Licht erlaubt keine eindeutigen Schlüsse, da Acetat zum Teil veratmet und ein Teil des respiratorischen $\mathrm{CO}_{2}$ sicher reassimiliert wird. Der Versuch wurde unter Bedingungen wiederholt, die einen wirksameren Austausch zwischen dem Kohlendioxyd der Gasphase und dem der Acetatveratmung zuzuschreibenden $\mathrm{CO}_{2}$ versprachen. Die Suspension wurde durch ein am Gefäßboden befindliches Glassinterfilter kräftig durchströmt. Das Atmungs- $\mathrm{CO}_{2}$ und somit auch das gebildete radioaktive $\mathrm{CO}_{2}$ sollte größtenteils im Luftstrom entfernt und somit eine Reassimilation weitgehend verhindert werden.
Beide Versuche, im Licht und im Dunkeln (Abb. 1) wurden nacheinander angestellt; die Daten sind also nur bedingt miteinander vergleichbar. Der Acetat-Einbau wird im Licht stark gefördert, sofern

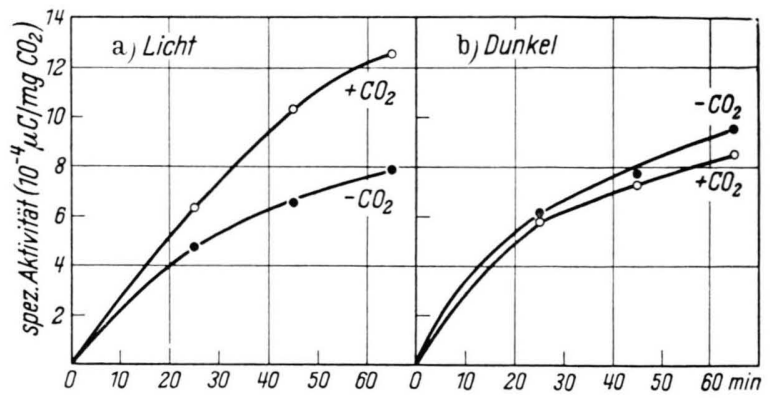

Abb. 1. Einbau von Acetat $-2 \cdot{ }^{14} \mathrm{C}$ in Chlorella unter kontinuierlicher Durchströmung mit $5 \% \mathrm{CO}_{2}$ enthaltender, bzw. durch $\mathrm{KOH}$ gewaschener Luft, im Licht (a) und im Dunkeln (b). Jedes Gefäß (Feinsinterfilter mit hohem Rand) enthielt $6 \mathrm{ml}$ Algensuspension (=61,2 mg Tr.-Gew.); $16 \mathrm{ml} 0,1-\mathrm{m}$. Phosphatpuffer $p_{\mathrm{H}} 5,6 ; 1 \mathrm{ml} 3 \cdot 10^{-3}-m$. $\mathrm{NaHCO}_{3}$, bzw. $\mathrm{H}_{2} \mathrm{O}$; $30 \mu$ Mole Acetat $(0,3 \mu \mathrm{C})$; seitliche Belichtung, Beleuchtungsstärke ca. $1000 \mathrm{fcdls}$. Proben von $5 \mathrm{ml}$ wurden 25, 45 und 65 min nach Zusatz des Acetats entnommen und mit $0,5 \mathrm{ml} 5-n \cdot \mathrm{H}_{2} \mathrm{SO}_{4}$ abgestoppt; Zellen zweimal gewaschen und spezifische Aktivität nach Verbrennung gemessen. Licht- und

Dunkelversuch wurden nacheinander unter den gleichen Bedingungen durchgeführt.

$\mathrm{CO}_{2}$ vorhanden ist. Im $\mathrm{CO}_{2}$-freien Milieu wird auch im Licht der Acetat-Einbau herabgesetzt. Die Depression der Acetat-Aufnahme durch $\mathrm{CO}_{2}$-Entziehung im Licht unter den Dunkeleinbau steht mit den Ergebnissen von Phillips ${ }^{14}$ im Einklang, nach denen das Wachstum von Chlorella mit Acetat bei $\mathrm{CO}_{2}$ Abwesenheit durch Licht gehemmt wird.

\section{Einbau von Acetat $-2 \cdot{ }^{14} \mathrm{C}$ in verschiedene Zellfraktionen}

Die Bestimmung des Quotienten $\Delta \mathrm{O}_{2} /-\Delta \mathrm{CO}_{2}$ während der Assimilation von $\mathrm{CO}_{2}$ und Essigsäure durch Chlorella pyrenoidosa hatte Werte bis zu 6 ergeben, woraus auf eine bevorzugte Synthese von Substanzen zu schließen war, die stärker reduziert sind als Kohlenhydrate; es wurde vermutet, daß unter den gegebenen Bedingungen Fette und Terpene aufgebaut werden. Um diese manometrischen Experimenten entstammenden Schlüsse in einer mehr direkten Weise nachzuprüfen, wurden Chlorella-Suspensionen im Licht und im Dunkeln in Gegenwart von markiertem Acetat geschüttelt; in gewissen Zeitintervallen wurden Proben entnommen, von denen die spezifischen Aktivitäten der in 


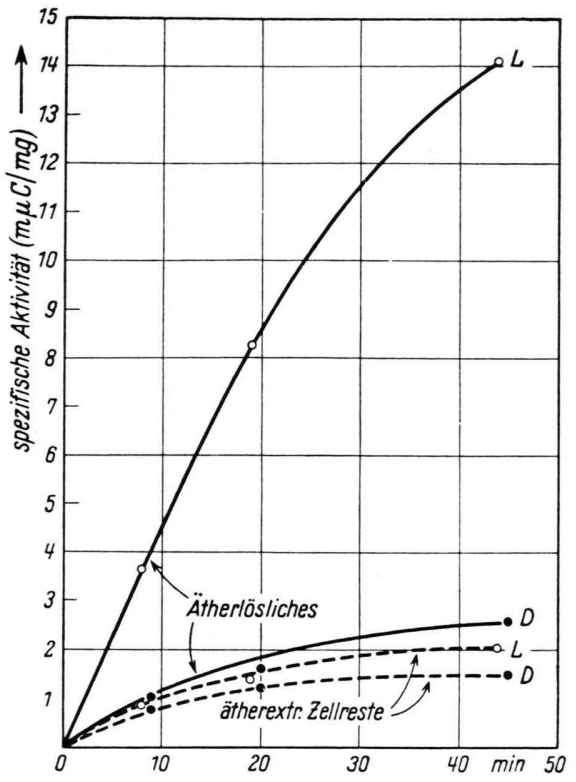

Abb. 2. Spezifische Aktivitäten im Ätherlöslichen und in den äther-extrahierten Zellresten von Chlorella als Funktion der Einbauzeit von Acetat-2-14 C. Je 0,5 g (Tr.-Gew.) ChlorellaZellen in $75 \mathrm{ml} m / 30$-Phosphatpuffer $p_{\mathrm{H}} 5,6$ wurden im Licht (2000 fcdls) und im Dunkeln geschüttelt; 9,20 und $45 \mathrm{~min}$ nach Zusatz von je $8 \mu$ Molen Acetat $-2 \cdot{ }^{14} \mathrm{C}$ wurden Proben entnommen und aufbereitet wie im Text angegeben. Spezifische Aktivität in $\mathrm{m} \mu \mathrm{C} / \mathrm{mg}$ des Petrolätherlöslichen, bzw. der äther-extrahierten Zellen.

Fettlösungsmitteln löslichen Komponenten und der extrahierten Zellreste bestimmt wurden. Einzelheiten der Versuchsdurchführung sind im methodischen Teil gegeben.

Abb. 2 gibt die Zunahme der spezifischen Aktivitäten im petroläther-löslichen Anteil und den ätherextrahierten Zellresten während der Assimilation von Acetat- $2 \cdot{ }^{14} \mathrm{C}$ im Licht und im Dunkeln wieder. Die spezifische Aktivität des Ätherlöslichen steigt im Licht etwa achtmal rascher an als im Dunkeln.
Während Licht den Einbau von Essigsäure in die äther-unlöslichen Komponenten der Zellen kaum erhöht, fördert es den Einbau in die Lipide außerordentlich. Die vorangegangenen Beobachtungen über die Erhöhung des Assimilationsquotienten während der Acetatassimilation im Licht berechtigen dazu, das Anwachsen der spezifischen Aktivität der Fettfraktion mit einer Netto-Synthese von Fetten zu interpretieren und nicht etwa nur als Ausdruck eines höheren "turnover" der Fette gegenüber anderen Zellbestandteilen zu werten.

Das Petrolätherlösliche wurde verseift und in Unverseifbares und Fettsäuren getrennt (Tab. 2). Da die spezifische Aktivität des Unverseifbaren, welches die Polyisoprenoide enthält, fragwürdig klein erschien, wurde $\beta$-Carotin chromatographisch isoliert und seine spezifische Aktivität bezüglich der durch Wägung wie auch der durch Extinktionsmessung bestimmten Menge errechnet; beide Werte stimmten gut überein. Glucose wurde durch Säurehydrolyse der in den fettextrahierten Zellen enthaltenen Stärke gewonnen und durch wiederholte Papierchromatographie gereinigt. Betrachten wir zunächst die Verhältnisse beim Einbau im Licht. Von den zugesetzten $21 \mu \mathrm{C}$ wurden $10,1 \mu \mathrm{C}$ in die petroläther-lösliche Fraktion eingebaut, die nahezu ein Drittel des Trokkengewichtes ausmacht. Davon fanden wir $6,2 \mu \mathrm{C}$ in der Fettsäurefraktion, die sicher nicht quantitativ erfaßt wurde. Diesen Beträgen gegenüber fallen die in die Fraktion des Unverseifbaren und in die Stärke eingebauten Mengen radioaktiven Acetats kaum ins Gewicht. In den spezifischen Aktivitäten der isolierten Fraktionen kommt der bevorzugte Einbau des zugesetzten Acetats in die Fraktion der Fettsäuren noch deutlicher zum Ausdruck. Die früher geäußerte Vermutung, daß Acetat im Licht auch bevorzugt in Terpenkörper eingeht, hat sich nicht bewahrheitet;

\begin{tabular}{|c|c|c|c|c|}
\hline \multirow{2}{*}{ Zellfraktion } & \multicolumn{2}{|c|}{$\begin{array}{c}\text { Gesamtaktivität } \\
{[\mathrm{m} \mu \mathrm{C}]}\end{array}$} & \multicolumn{2}{|c|}{$\begin{array}{l}\text { Spezifische Aktivität } \\
{[\mathrm{m} u \mathrm{C} / \mathrm{mgC}]}\end{array}$} \\
\hline & Licht & Dunkel & Licht & Dunkel \\
\hline $\begin{array}{l}\text { Petrolätherlösliches } \\
\text { davon in Fettsäuren } \\
\text { davon im Unverseif baren } \\
\text { davon in } \beta \text {-Carotin } \\
\text { Glucose (hydrolys. Stärke) }\end{array}$ & $\begin{array}{r}10100 \\
6200 \\
240 \\
6 \\
73\end{array}$ & $\begin{array}{c}1320 \\
760 \\
47 \\
1,3 \\
68\end{array}$ & $\begin{array}{r}104 \\
108 \\
12 \\
11,2 \\
7,5\end{array}$ & $\begin{array}{r}14,3 \\
14,1 \\
2,5 \\
2,3 \\
6,9\end{array}$ \\
\hline
\end{tabular}

Tab. 2. Einbau von Acetat-2.14 C in verschiedene Zellfraktionen von Chlorella im Licht und im Dunkeln. Chlorellazellen (je $0,52 \mathrm{~g}$ Tr.-Gew.) in $100 \mathrm{ml} \mathrm{m} / 30$-Phosphatpuffer $p_{\mathrm{H}} 5,6$ unter $5 \% \mathrm{CO}_{2}$ Luft-Atmosphäre im Licht (2000 fcdls) bzw. Dunkelheit $30 \mathrm{~min}$ vorinkubiert, dann $21 \mu$ Mole $(=21 \mu \mathrm{C})$ Acetat $-2 \cdot{ }^{14} \mathrm{C}$ zugefügt und 20 min geschüttelt; Einbau beendet durch Zusatz von je $0,4 \mathrm{ml} \mathrm{1-n.} \mathrm{HCl}$ und Eiskühlung. Zellen wurden abzentrifugiert und gewaschen. In den vereinigten und bei $p_{\mathrm{H}} 9,0$ eingeengten überstehenden Lösungen wurde das unverbrauchte Acetat nach Wasserdampfdestillation bestimmt; im Licht wurden 0,28 und im Dunkeln 7,6 $\mu \mathrm{C}$ unverbraucht gelassen. 
ihre spezifische Aktivität, repräsentiert durch Werte für $\beta$-Carotin, liegt zwar noch über dem Wert für Glucose, beträgt aber nur ein Zehntel des Wertes für Fettsäuren.

Im Dunkeln wurden nur $1,32 \mu \mathrm{C}$ in das Petrolätherlösliche eingebaut. Die spezifischen Aktivitäten der Fettfraktionen sind beträchtlich geringer als im Licht. Aber auch hier liegen die Werte für Fettsäuren höher als für Polyisoprenoide und Glucose. Das bedeutet, daß das Acetat selbst im Dunkeln (unter den hier gegebenen Bedingungen des N-Mangels und nach Verarmung) vorzugsweise in Fettsäuren eingebaut wird.

Nach den vorliegenden Ergebnissen steigert Licht den Acetateinbau in Chlorella-Zellen in einer spezifischen Weise, indem es die Bildung von Speicherstärke nur unbedeutend stimuliert, die Synthese von Fettsäuren aus Acetat aber außerordentlich begünstigt. Es ist anzunehmen, daß dieser Effekt unter den hier angewandten Bedingungen des N-Mangels und nach Verarmung der Zellen noch ausgeprägter in Erscheinung tritt als unter Wachstumsbedingungen. Die Assimilation von markiertem Acetat durch gewaschene Suspensionen von Chlorella im Licht bietet sich als ein rentabler Weg zur Synthese markierter langkettiger (C 16 und C 18) Fettsäuren aus Essigsäure an.

\section{Is otopenverteilung im Glucosemolekül}

Die gereinigte Glucose wurde dem biologischen Abbau durch Leuconostoc mesenteroides unterwor- fen, und die spezifische Aktivität jedes einzelnen Kohlenstoffatoms des Glucosemoleküls erfaßt. In Tab. 3 sind die Ergebnisse des Abbaus von Glucose, die aus drei voneinander unabhängigen Versuchen stammt, dargestellt. Die Versuchsbedingungen weichen hinsichtlich der Einbauzeiten und eingesetzten Aktivitäten voneinander ab, wodurch die unterschiedlichen spezifischen Aktivitäten hinreichend erklärt werden. Dennoch stimmen die Relationen zwischen den gewonnenen Daten soweit überein, daß sie gemeinsam interpretiert werden können. Die im vorangehenden Abschnitt herausgestellte Tatsache, daß die im Dunkeln und die im Licht gebildete Glucose nahezu die gleiche spezifische Aktivität aufweist, kann bereits als Hinweis dafür angesehen werden, daß sich der Mechanismus der Stärkesynthese unter beiden Bedingungen nicht nennenswert unterscheidet. Die gewonnenen Werte bestätigen diese Annahme. Um eine rasche Orientierung zu ermöglichen, wurden die Mittelwerte der relativen spezifischen Aktivitäten $(\mathrm{C} 6=100)$ in Abb. 3 graphisch dargestellt.

Zunächst sei die vorherrschende Symmetrie in der Markierung der Kohlenstoffatome der Glucosekette (C $1=2=5=6>$ C $3=4)$ kurz erörtert. Diese Isotopenverteilung im Glucosemolekül deutet auf die maßgebliche Beteiligung des Tricarbonsäurezyklus (TCC) bei der Assimilation von Essigsäure durch Chlorella. Der TCC in seiner bisherigen Form ist jedoch nur für dissimilatorische Prozesse von Bedeutung; für jeden in den Zyklus einmündenden C 2Körper werden zwei Moleküle Kohlendioxyd abgespalten. Nennenswerte synthetische Leistungen

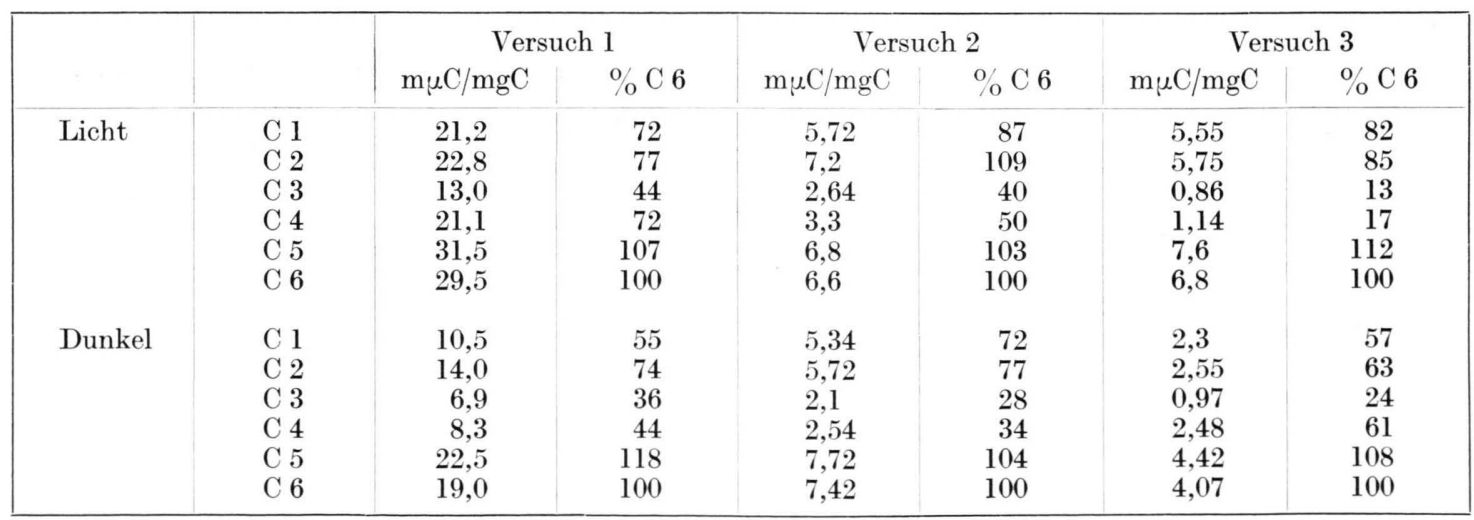

Tab. 3. Verteilung von ${ }^{14} \mathrm{C}$ im Glucosemolekül. Vers. 1. 0,5 g (Tr.-Gew.) Chlorella; $30 \mu$ Mole Acetat; Einbauzeit 35 Minuten. Die mit 80-proz. wäßrigem Äthanol extrahierten Zuckerester wurden gemeinsam mit den Zellen hydrolysiert. Vers. 2. Circa 0,4 g (Tr.-Gew.) Chlorella; $2 \mu$ Mole Acetat; Einbauzeit 30 Minuten. Die Glucose wurde durch Säurehydrolyse der fettextrahierten Zellen gewonnen. Vers. 3. 0,72 g (Tr.-Gew.) Chlorella; $25 \mu$ Mole Acetat, Einbauzeit 20 Minuten. Die Glucose wurde durch Säurehydrolyse der fettextrahierten Zellen gewonnen. 
kann man demnach mit der Funktion des TCC nicht erklären. Nach der Entdeckung der IsocitrataseReaktion ${ }^{15}$ und der Äpfelsäure-Synthese aus Glyoxylsäure und Acetyl-Coenzym A ${ }^{16}$ haben Kornberg und Krebs ${ }^{17}$ daher auf die Beteiligung dieser Reak-

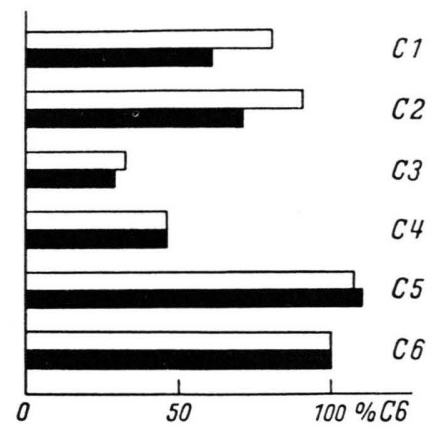

Abb. 3. Verteilung von ${ }^{14} \mathrm{C}$ über das Glucosemoleköl. Mittel der relativen, spezifischen Aktivitäten der im Licht, bzw. im Dunkeln synthetisierten Glucose (helle bzw. schwarze Rechtecke).

tionen an der Synthese von Zellsubstanzen aus Essigsäure hingewiesen und die sich unter Berücksichtigung dieser Reaktionen ergebende Modifikation des TCC wegen der Beteiligung der Glyoxylsäure „Glyoxylsäure-Zyklus" genannt. Dabei tritt Essigsäure an zwei Stellen in den Zyklus ein. Die hervorragende Bedeutung dieses Zyklus für alle mit Acetat und "acetogenen“ Substraten wachsenden Organismen und für die Umwandlung von Fetten in Kohlenhydrate ${ }^{18}$ ist unmittelbar einzusehen. Die Interpretation der Isotopenversuche erfährt durch die Substitution dieses „Glyoxylsäure-Zyklus“ für den TCC in qualitativer Hinsicht keine Revision, da sowohl im TCC als auch im Glyoxylsäure-Zyklus der Methyl-C der Essigsäure in die beiden mittleren C-Atome der C 4-Säuren eingebaut und die durch deren Decarboxylierung gebildete C 3-Säure lediglich in den NichtCarboxyl-C-Atomen markiert wird. Das Isotopenmuster in der synthetisierten Glucose folgt dann aus Reaktionen der Glykolyse: Glycerinaldehyd-3-phosphorsäure (GA-3-P) liefert die C-Atome 4, 5 und 6 und die mit Glycerinaldehyd-3-phosphorsäure im

14 J. N. Phillips, zitiert nach J. Myers, Annu. Rev. Microbiol. 5, 157 [1951].

15 R. S. Smith u. I. C. Gunsalus, J. Amer. chem. Soc. 76, 5002 [1954]; R. S. Sмith u. I. C. Gunsalus, J. biol. Chemistry 229, 305 [1958].

${ }^{16}$ D. T. O. Wong u. S. J. AJl, J. Amer. chem. Soc. 78, 3230 [1956].

17 H. L. Kornberg u. H. A. Krebs, Nature [London] 179, 988 [1957].
Triosephosphatisomerase-Gleichgewicht stehende Dihydroxyaceton-phosphorsäure (DHA-P) liefert die C-Atome 1, 2 und 3. Eine prinzipiell gleiche Isotopenverteilung wurde im Glucosemolekül der Speicherkohlenhydrate von verschiedenen Organen und Organismen nach Verfütterung von Acetat- $2 \cdot{ }^{14} \mathrm{C}$ gefunden, für die durch enzymchemische Studien die Funktion des TCC und der Glykolyse gut fundiert ist. Die vorgelegten Ergebnisse stützen somit die Vorstellung, daß diese Prozesse auch in Chlorella pyrenoidosa die dominierenden Routen des Intermediärstoffwechsels darstellen.

Als zweiter hervorstechender Zug der Isotopenverteilung im Glucosemolekül bedarf die Asymmetrie $\mathrm{C} 4+5+6>\mathrm{C} 1+2+3$ einer Erörterung. Es handelt sich hierbei um eine verschiedentlich gemachte Beobachtung, die man mit einem ungenügenden Triosephosphatisomerase-Gleichgewicht und der Verdünnung der DihydroxyacetonphosphorsäureEinheit durch einem "pool“ entstammende Bausteine interpretieren könnte ${ }^{19}$. Es braucht sich dabei keineswegs um einen pool von Glycerin oder Dihydroxyacetonphosphorsäure zu handeln: durch das Enzym Transaldolase, das gerade im Stoffwechsel photosynthetischer Organismen eine wesentliche Rolle spielt ${ }^{20,21}$, wird die Verbindung zum pool der Intermediärprodukte des Photosynthesezyklus hergestellt. Transaldolase überträgt Dihydroxyaceton von Sedoheptulose-7-phosphat oder Fructose-6phosphat auf Glycerinaldehyd-3-phosphorsäure. Aldolase überträgt Dihydroxyacetonphosphorsäure von Sedoheptulose-1,7-diphosphat auf Glycerinaldehyd-3-phosphorsäure. In unserem Falle dürfte im Zuge dieser Übertragungsreaktionen ein Teil der markierten Glycerinaldehyd-3-phosphorsäure mit unmarkierten Dihydroxyaceton-phosphat zusammentreten und so Glucosemoleküle entstehen lassen, in denen lediglich die C-Atome 4,5 und 6 markiert sind. In diesen Reaktionen findet die beobachtete Asymmetrie ein einfache Erklärung.

Die einfachste Erklärung für die Ungleichheit der spezifischen Aktivitäten der C-Atome in 1,2 und 5,

18 H. L. Kornberg u. H. Beevers, Nature [London] 180, 35 [1957].

19 P. Schambye, H. G. Wood u. G. Popjak, J. biol. Chemistry 206, 875 [1954].

20 B. L. Horecker u. P. Z. Smyrniotis, J. biol. Chemistry 212. 811 [1955].

21 W. Vishniac, L. B. Horecker u. S. Ochoa, Advances in Enzymol. 19, 1 [1957]. 
6-Stellung ist in einer teilweisen minimalen Gleichgewichtseinstellung mit einem symmetrischen C 3 Körper zu sehen. So könnte durch Phosphatasewirkung aus Dihydroxyacetonphosphorsäure Dihydroxyaceton entstehen, das durch Glycerokinase und ATP wieder zu Dihydroxyacetonphosphorsäure rephosphoryliert wird ${ }^{22,23}$, wobei die Phosphorylierung an beiden Enden des symmetrischen Moleküls mit der gleichen Wahrscheinlichkeit erfolgen sollte. Durch diese Reaktionen würde auf der Stufe der Dihydroxyacetonphosphorsäure eine teilweise Herabsetzung der spezifischen Aktivität von C 1 erfolgen, was sich durch Rückreaktionen auch auf C 6 auswirken könnte. Die spezifische Aktivität der C-Atome 3 und 4 würde dadurch natürlich heraufgesetzt.

\section{Diskussion}

Daß Essigsäure und wahrscheinlich auch andere organische Säuren im Licht bevorzugt in Fettsäuren eingebaut werden, erscheint in Anbetracht der laufenden Regeneration von reduzierten Pyridinnucleotiden unter diesen Bedingungen plausibel. Im Stoffwechsel der Zelle scheint der Prozeß am meisten gefördert zu werden, welcher der im Überschuß produzierten Reduktionskraft am meisten bedarf, also die Fettsynthese gegenüber der Synthese von Kohlenhydraten. Daß die Kohlenwasserstoffe, insbesondere die Carotinoide von dieser bevorzugten Synthese ausgeschlossen sind, obwohl auch sie über Acetyl-Coenzym A aufgebaut werden, mag auf den Umstand zurückzuführen sein, daß diese Substanzen keine natürlichen Speicherstoffe von Chlorella darstellen.

Die Intermediärprozesse, die zu der angegebenen Isotopenverteilung über die C-Atome des Glucosemoleküls führen, wurden bereits im experimentellen Teil diskutiert. Lediglich die asymmetrische Markierung soll hier im Zusammenhang mit einigen Befunden besprochen werden, die in den letzten Jahren beim Studium der Kohlenhydrat-Synthese tierischer Organe erhoben wurden. Bei langfristigen Einbauversuchen mit Ratten fanden Woop und Mit-

22 C. Bublitz u. E. P. Kennedy, J. biol. Chemistry 211, 951 [1954].

23 O. Wieland u. M. Suyter, Biochem. Z. 329, 320 [1957].

24 P. Schambye, H. G. Wood u. M. Kleiber, J. biol. Chemistry 226, 1011 [1957].

25 P. A. Marks u. B. L. Horecker, J. biol. Chemistry 218, 327 [1956]. arbb. stets eine symmetrische Verteilung der Isotope entlang der Glucosekette des Leberglykogens. Erst die Anwendung der biologischen Degradationsmethode mit Leuconostoc mesenteroides, die die routinemäßige Bestimmung der spezifischen Aktivität jedes einzelnen Kohlenstoff-Atoms im Glucosemolekül ermöglicht, lenkte die Aufmerksamkeit auf eine gewisse Asymmetrie im Isotopenmuster. Eine solche wurde zuerst für Glucose aus Leberglykogen beschrieben ${ }^{19}$, das aus Ratten nach Verfütterung von Glycerin $-{ }^{14} \mathrm{C}$ isoliert worden war: in $\mathrm{C} 3$ wurde eine 1,4-2,4-fach höhere Aktivität gefunden als in C 4. Ein umgekehrtes Verhältnis (C 4>C 3) fanden Schambye, Wood und Kleiber ${ }^{24}$ in der Glucose und Galaktose des Milchzuckers aus Milch nach intravenöser Injektion der Kuh mit Acetat-1-14C. Von besonderem Interesse sind in diesem Zusammenhang die Ergebnisse von Marks und Horecker ${ }^{25}$, die Leberschnitte von normal gefütterten und $72 \mathrm{Stdn}$. lang ausgehungerten Ratten mit ${ }^{14} \mathrm{CO}_{2}$ und Milchsäure inkubierten; in letzteren war die isolierte Glykogen-Glucose symmetrisch, in ersteren hingegen unsymmetrisch (C 4>C 3) markiert. Wurden die Hunger-Ratten-Leberschnitte mit Lactat $+{ }^{14} \mathrm{CO}_{2}+\mathrm{Gly}$ cerin inkubiert, so wurde auch hier eine ausgeprägte Asymmetrie aufgefunden. Die skizzierten Resultate verschiedenartiger Einbauversuche stehen mit der Vorstellung im Einklang, daß Glucose über Reaktionen der Glykolyse aus GA-3-P $(\mathrm{C} 4,5,6)$ und DHA-P (Cl, 2, 3 der Glucose) synthetisiert wird. Während Glycerin in die glykolytischen Reaktionen über DHA-P eintritt, münden Milchsäure, Brenztraubensäure, 3-Phosphoglycerinsäure, Essigsäure und alle Verbindungen, die Acetyl-Coenzym A bilden können („,acetogene Substrate“), über GA-3-P in die glykolytischen Reaktionen ein.

In vivo ist stets ein gewisser pool vorhanden, durch den die zugesetzten isotopenhaltigen Verbindungen verdünnt werden ${ }^{*}$; die Verteilung der Isotopen über das Glucosemolekül hängt dann davon ab, ob das zugesetzte radioaktive Substrat über DHA-P oder GA-3-P in die glykolytischen Reaktionen eingeschleust wird und der pool groß oder klein ist. Außerdem wird der Grad der Asymmetrie um so

\footnotetext{
* Auf die Möglichkeit, daß die Verdünnung der Dihydroxyacetonphosphat-Einheit durch einen aus Glycerophosphaten gespeisten pool erfolgt, weist auch Besson hin. (A. A. Benson, Proc. Int. Symp. Enzyme Chemistry, Tokyo 1957, $189-191$.)
} 
größer sein, je kürzer die Einbauzeit gehalten wird. Daß mit der Speisung des pools an DHA durch Transaldolase-Reaktionen aus dem pool der Intermediärprodukte des Pentosephosphatzyklus zu rechnen ist, wurde bereits erwähnt.

Die asymmetrische Markierung der Hexosen aus Chlorella nach kurzfristiger ${ }^{14} \mathrm{CO}_{2}$-Fixierung ${ }^{3,26}$ findet durch die vorgelegten Befunde keine Erklärung. Es ist lediglich darauf hinzuweisen, daß auch Sedoheptulose-7-phosphat und Fructose-6-phosphat als Quelle von Dihydroxyaceton anzusehen sind. Wird DHA von Zuckerphosphatestern, die den Pentose- phosphatzyklus schon mehr als zweimal durchlaufen haben, durch Transaldolase auf GA-3-P übertragen, so kommt es zu einer Isotopenverteilung, wie sie von den Autoren gefunden wurde: C $4>\mathrm{C} 3$ und C 1, 2>C 5, 6. Auch die Beobachtung, daß durch zusätzliche Verabreichung von Glucose $\mathrm{zu}$ den ${ }^{14} \mathrm{CO}_{2}$ fixierenden Zellen die Asymmetrie über längere Einbauzeiten erhalten bleibt, findet in der Annahme eines durch Glucose gespeisten pools eine hinreichende Erklärung.

26 O. Kandler u. M. Gibbs, Plant Physiol. 31, 411 [1956].

\title{
Manometrische Messungen des respiratorischen Stoffwedhsels von Gewebekulturen aus Affennieren-Zellen unter besonderer Berücksichtigung einiger physiologischer Momente
}

\author{
Von Th. Luthardt, R. Sauthoff und H. K. Mittelstrass* \\ Aus der Universitäts-Kinderklinik Freiburg i. Br. (Direktor: Prof. Dr. W. Keller) \\ (Z. Naturforschg. 14 b, 253-259 [1959]; eingegangen am 22. Dezember 1958)
}

\begin{abstract}
In vitro kultivierte Affennieren-Zellen geben unter physiologischen Meßbedingungen keinen Anhaltspunkt für die Entwicklung eines Gärungsstoffwechsels im Sinne einer Krebsentstehung. Ausgewachsene Kulturen weisen nach Mediumerneuerung zunächst aerobe Glykolyse auf, diese geht im Laufe eines Tages praktisch vollkommen zurück, der oxydative Stoffwechsel ändert sich währenddessen nicht; Messungen über drei weitere Tage geben keine Änderungen dieses Bildes mehr. Das Auftreten eines Gärungsstoffwechsels in Suspensionen von in vitro kultivierten Nierenzellen und bei ausgewachsenen Kulturen unmittelbar nach Mediumerneuerung ist kein Anzeichen für die Entstehung eines Krebsstoffwechsels; wahrscheinlich handelt es sich u. a. dabei um einen vorübergehenden durch das neue Medium bedingten Wachstums-Stoffwechsel.
\end{abstract}

Stoffwechseluntersuchungen, gleich welcher Art, an Gewebekulturen in vitro zeichnen sich durch methodische Kompliziertheiten aus, die zweifacher Natur sind. Zu den empfindlichen Meßmethoden kommt die Schwierigkeit, die Kulturen während der Messung in gutem Zustand zu halten; nur zu leicht unterliegt man diesen Schwierigkeiten und mißt unter unphysiologischen Bedingungen an nicht repräsentativem Material.

Manometrische Messungen des Stoffwechsels von Kartoffel-Gewebeschnitten, an denen einer von uns beteiligt war, zeigten, daß es nach Änderung von Umgebungsbedingungen bis zu Tagen dauert, ehe ein neues Stoffwechsel-Gleichgewicht erreicht wird ${ }^{1-3}$. Suschny, Kellner, Broda, Figdor und Rücker ${ }^{4}$

\footnotetext{
* Diese Arbeit wurde aus Mitteln der D e u t s c he n F or . $\mathrm{schungs}$ gem ein s c h a f t gefördert.

1 J. Pichotka, Med. meteor. Hefte 13, 38 [1958].

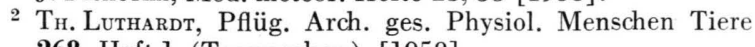
268, Heft 1 (Tagungsber.) [1958].
}

haben auch für die in vitro-Gewebekultur menschlicher Gewebe im Rahmen ihrer Messungen mit radioaktiven Isotopen auf diesen Faktor ausführlich hingewiesen. Fermentuntersuchungen in verschiedenen Gewebekultur-Fraktionen, z. B. in den Zellen, im Kulturüberstand, in der Versenfraktion beim Versenieren, wurden von Matzelt und HomanN ${ }^{5}$ durchgeführt; sie zeigen ebenfalls sehr deutlich, wie leicht das Gleichgewicht von Gewebekulturen gestört wird, und $d a ß$ es viele Stdn. braucht, bis wieder konstante Verhältnisse vorliegen. Ebenso zeigt der zeitliche Verlauf beim Glucoseeinbau in Gewebekulturen nach Änderung von Umgebungsbedingungen, etwa nach Mediumwechsel, über viele Stdn. sich ändernde Werte $^{6}$, das gleiche gilt für die Milchsäureproduk-

3 J. Pichotka, W. Höfler u. Th. Luthardt, in Vorbereitung.

4 O. Suschny, G. Kellner, E. Broda, B. Figdor u. W. Rücker, Exp. Cell Res. 14, 316 [1958].

5 D. Matzelt u. J. Homann, Biochem. Z. 330, 245 [1958].

${ }_{6}$ Y. Becker, N. Grossowicz u. H. Bernkopf, Proc. Soc. exp. Biol. Med. 97, 77 [1958]. 